\title{
Diminished Ovarian Reserve, Causes, Assessment and Management
}

\author{
Mandeep Kaur, Mala Arora
}

\section{ABSTRACT}

Diminished ovarian reserve predicts diminished ovarian response to stimulation but does not predict cycle fecundity. It has been recently defined by ESHRE, the Bologna's criteria, according to which at least two of the following three features should be present: (1) Age $>40$ years/any other risk factor for DOR, (2) abnormal ovarian reserve test, i.e. antral follicle count, $\mathrm{AMH},(3)$ poor ovarian response in a previous stimulated cycle, i.e. less than three follicles after standard gonadotropin stimulation. Poor response to maximal stimulation on two previous occasions also defines DOR.

The treatment options are limited. Avoiding the GnRH agonist long protocol and stimulation with microdose flare or antagonist protocol yields better results. Adjuvant therapy with $\mathrm{LH}$, DHEAS and growth hormone shows some benefit in improving the oocyte yield. It is advisable to perform ICSI for all obtained oocytes and some advocate assisted hatching. Pregnancy rates are, however, poor and often these patients require ovum donation. Developing tests that will diagnose DOR in a low-risk population will allow women to plan their reproductive careers early.

Keywords: Diminished ovarian reserve, Poor ovarian response, Premature ovarian failure, Poor responders.

How to cite this article: Kaur M, Arora M. Diminished Ovarian Reserve, Causes, Assessment and Management. Int J Infertility Fetal Med 2013;4(2):45-55.

Source of support: Nil

Conflict of interest: None

\section{INTRODUCTION}

The term ovarian reserve (OR) is a term bound for confusion. The term signifies the magnitude of ovarian response in assisted reproductive treatment (ART) cycles not the fecundity in a natural cycle. As of date, there is no accepted definition of 'diminished' ovarian reserve (DOR) and it may refer to three related but different parameters:

- The oocyte quantity

- The oocyte quality

- The oocyte reproductive potential.

Ovarian reserve may be defined as the number and quality of the remaining follicles and oocytes in both the ovaries at a given age. Decline in the number of follicles below a threshold level leads to irregular cycles and ultimately menopause; decline in their quality results in poor

Date of Received: 17-08-2013

Date of Acceptance: 25-08-2013

Date of Publication: May 2013 reproductive outcome. Human oocytes peak in number during fetal life reaching a maximum number of 6 to 7 million at 20 weeks. The process of atresia also starts simultaneously. At birth, the number available is about 1 to 2 million. After puberty, with each ovulatory cycle, a cohort of follicles get selected, one of them grows as the dominant follicle and rest undergo apoptosis. The atrophied follicles can not regenerate. As the number of primordial follicles get depleted, the antral follicle count declines. The decline in number seems to be rapid after less than 25,000 primordial follicles remain.

Female fertility declines with age but it is difficult to predict the pace of reproductive decline in an individual woman. With the postponement of childbearing, especially in western countries, the rate of subfertility related to female age has increased considerably. ${ }^{1}$ Female fecundity declines after the age of 31 years and the decline accelerates after the age of 37 years leading to sterility at the age of 41 years. ${ }^{2}$ The rate of decline varies considerably between females of the same age. The human species are relatively subfertile with a monthly fecundity of about $20 \%$. The proportion of subfertility is 10 to $20 \%$ of couples with females aged 35 years but only $4 \%$ in women in their 20 s. However, the maintenance of regular cycles till the fertility potential has reduced to almost zero makes many females unaware of this natural decline in reproductive potential.

Diminished ovarian reserve (DOR) is used to describe women of reproductive age with regular cycles, mostly ovulatory, whose response to ovarian stimulation is reduced compared to women of comparable age. It is distinct from menopause or premature ovarian failure where the cycles are anovulatory and irregular, and levels of FSH are raised. Diminished ovarian reserve (DOR) should probably replaced with the term diminished ovarian response (DOR). It is also sometimes described as primary ovarian insufficiency. ${ }^{3}$

Early loss of ovarian function has significant psychosocial sequelae due to poor reproductive performance but also has major health implications. ${ }^{4}$ Nearly, two-fold age-specific increase in mortality rate has been reported ${ }^{5}$ in patients with premature menopause.

\section{CAUSES OF DOR}

In most cases, the causes of DOR are unknown. It is unclear whether DOR represents a pathologic condition resulting 
from abnormally rapid atresia in a normal pool of oocytes, from normal atresia of an abnormally small initial pool of oocytes or simply the extreme end of a normal bell-shaped population distribution of the number of oocytes at a given age. The causes of diminished ovarian reserves are enumerated in Table 1.

Chemotherapy normally targets actively dividing cells and might not cause an adverse effect on the oocyte. Chemotherapy causes depletion of the primordial follicles in a drug and a dose dependant manner. Alkylating agents can affect both resting and dividing cells as its action is not cell cycle dependent. Risk of damage is more with advancing age; however, individual women differ in their sensitivity to gonadal damage from the same drug and in a similar dosage. Radiation therapy damage on the ovaries depends on the age of the patient, radiation dose and the radiation field. There may be recovery in a period of 6 to 18 months or irreversible damage especially in older patients. The prepubertal ovary is relatively resistant to this form of gonadotoxicity. ${ }^{6}$ Transposition of the ovaries in young women requiring pelvic irradiation helps in preserving their ovarian function.

Fragile $\mathrm{X}$ syndrome is the most common cause of inherited mental retardation and autism and it occurs due to abnormal expansion of CGG repeats in the FMR1 (fragile $\mathrm{X}$ mental retardation gene) located on the long arm of $\mathrm{X}$-chromosome. Normally, there are 30 repeats but, in premature ovarian failure, there could be 50 to 200 repeats. Women with premutations exhibit early ovarian ageing and early menopause. There may be transmission to the offspring as the length of sequence is unstable and may expand when transmitted from mother to offspring. Another genetic cause of decreased ovarian reserve is $45 \mathrm{X}$ genotype (Turner syndrome) and its mosaic, in which oocyte loss is accelerated after 18 weeks in utero. ${ }^{7}$

Menopause can occur 1 to 4 years earlier in smoking women than nonsmokers. ${ }^{8}$ Chemicals in cigarette smoke appear to accelerate follicular depletion and the loss of

Table 1: Causes of decreased ovarian reserve

1. Exposure to chemotherapy

2. Exposure to radiotherapy

3. Genetic abnormalities (45X, FMR-premutations)

4. Smoking

5. Previous ovarian surgery

6. Uterine artery embolization and ligation

7. Autoimmune disorders, like Addison's disease

8. Galactosemia

9. Mumps oophoritis

10. Salpingectomy for ectopic, hydrosalpinx, etc.

11. Environmental factors

12. FSH receptor polymorphisms

13. Idiopathic reproductive function. Mean basal follicle-stimulating hormone (FSH) levels are significantly higher in young smokers than in nonsmokers. ${ }^{9}$ Urinary estrogen excretion during the luteal phase in smokers is only about one-third that observed in non-smokers, possibly because constituents of tobacco smoke inhibit granulosa cell aromatase. ${ }^{10}$ Current smoking is also associated with lower anti-mullerian hormone $(\mathrm{AMH})$ levels; mean gonadotropin dose requirements for smokers receiving stimulation for in vitro fertilization (IVF) are higher when compared with those of nonsmoking women. ${ }^{8}$

A significant adverse event following uterine artery embolization (UAE) is diminished ovarian reserve or premature ovarian failure, particularly in older women. Successful pregnancies after UAE have been described, but data relating to fertility and pregnancy outcomes after UAE are still quite limited, and the procedure therefore cannot be recommended for women planning pregnancy later. ${ }^{11}$ Uterine artery ligation for the management of postpartal hemorrhage may also decrease the ovarian reserves by affecting the blood supply of the ovaries.

Ovarian failure is slightly more common in women with insulin-dependent diabetes mellitus, myasthenia gravis and parathyroid disease than in healthy women. ${ }^{12}$ Autoimmune lymphocytic oophoritis may be seen in Addison's disease. Ovarian biopsy is not indicated in clinical practice, but because autoimmune ovarian failure could be a component of a polyglandular syndrome, patients can be screened for other abnormalities by means of thyroid stimulating hormone (TSH), thyroid autoantibodies, fasting glucose and electrolytes. ${ }^{13}$

Classic galactosemia is a rare cause of ovarian failure. Intracellular accumulation of galactose metabolites or deficient glycosylation reactions could lead to decrease in the initial number of oogonia through apoptosis. ${ }^{14}$ It is also suggested that the impact of galactosemia on the ovary could be due to the absence of recognition of circulating FSH by its receptor and not to a toxic alteration of the ovary by itself. ${ }^{15}$ Mumps oophoritis has been considered to be a cause of decreased ovarian reserve and POF. ${ }^{16}$ However most of the time, these conditions are associated with premature ovarian failure rather than DOR.

Laparoscopic salpingectomy is done by coagulating and dividing the proximal tube close to the cornua. The mesosalpinx is then serially coagulated and cut. It is prudent to stay close to the tube to avoid compromising the ovarian blood supply. Salpingectomy and even tubal ligation have been implicated as causes of subsequent diminished ovarian reserve, if the ovarian blood supply is compromised. However, several studies have been done that disprove this 
proposition. ${ }^{17,18}$ This may be due to varying effects of the procedure on ovarian blood supply.

The effects of endocrine disruptors, heavy metals, solvents, pesticides, plastics and industrial chemicals on female reproduction has been reviewed. ${ }^{19}$ The mechanism by which chemicals affect ovarian function may involve hormonal or immune disruption, DNA adduct formation, altered cellular proliferation, or inappropriate cellular death. Data on the association of chemical exposures and adverse reproductive outcomes in humans are, however, equivocal and further studies are needed to clarify which toxicants affect human reproduction and how.

Mutations of the FSH receptor gene were found to be rare in women with ovarian failure which is also known as resistant ovary syndrome or Savage syndrome. FSHR genes in patients with ovarian failure revealed the existence of polymorphisms which do not appear to have pathophysiological significance with regard to ovarian function. ${ }^{20}$

In idiopathic cases, there may be involvement of as yet unknown mechanisms affecting the rate of oocyte apoptosis. ${ }^{21}$ This may lead to a reduced complement of oocytes in the ovaries at birth or accelerated atresia. According to Barkers, hypothesis** the exposure to endocrine disrupters to a female fetus in utero may result in DOR later in life.

\section{ASSESSMENT OF OR}

Ovarian reserve (OR) tests are aimed to help predict ovarian reserve and/or reproductive performance in stimulated cycles. However, the presently available tests have many limitations, as they do not predict fecundity in a natural cycle. In women with regular menses, ovarian reserve tests do not predict whether they are entering menopause or whether they are experiencing a decline in fertility that is pathological. The goal of ovarian reserve testing is to add more prognostic information to the counseling and planning process so as to help couples choose among treatment options. However, it is important to emphasize that ovarian reserve tests should not be the sole criteria used to deny patients access to assisted reproductive technologies (ART) or other treatments. Evidence of decreased ovarian reserve is not equivalent to inability to conceive.

Ovarian reserve can be considered normal in conditions where ovarian stimulation with gonadotropins results in development of 5 to 13 follicles and there is retrieval of equivalent number of oocytes following follicle puncture. ${ }^{22}$ With such a yield, the chances of producing live birth with IVF are considered optimal. A mean oocyte number of 13.1 was seen to be associate with highest pregnancy rate per embryo transfer and per started cycle. ${ }^{23}$ Ovarian reserve tests also determine the follicular sensitivity to gonadotropins and determine the dose, especially in young patients liable to hyper-response.

Ovarian reserve tests can predict reduced fecundability and poor response to stimulation but this cannot be used to directly predict clinically important outcomes like clinical pregnancy and live birth. These can be used as screening tests but cannot make a definitive diagnosis. Ovarian reserve tests can predict ovarian response but cannot predict a live birth as it is affected by a number of factors like laboratory performance and transfer technique. The ovarian reserve tests should be highly specific, so as the number of false positives decreases. The advantages of performing OR tests are shown in Table 2.

Positive and negative predictive values are characteristics of screening tests that change with the prevalence of disease in a population. These are important in deciding whom to screen. If prevalence of disease in a population is low, PPV will be low even if the sensitivity or specificity is high. Thus, it is very important to understand that ovarian reserve testing is very useful in patients at high risk of DOR. The use of these tests in a low-risk population will cause women with normal reserves at risk for being categorized as DOR. The various available ovarian reserve tests are discussed in Table 3.

Table 2: Advantages of performing ovarian reserve tests

Advantages and disadvantages of highly specific ovarian reserve tests:

1. Patients with normal reserves are not wrongly categorized as DOR.

2. Avoids aggressive treatment in normal patients.

3. Prevents recommendation of donor oocytes in normal patients.

4. More women will attempt treatment with own gametes even when the prognosis is poor.

Table 3: Ovarian reserve tests (ORT)

\begin{tabular}{ll}
\hline Clinical & - Age \\
& - Menstrual cycle characteristics \\
\hline Biochemical & B Basal FSH \\
& Basal E2 \\
& - AMH \\
& Inhibin B \\
\hline Ultrasonographic & - Antral follicle count (AFC) \\
& - Ovarian volume \\
& Ovarian blood flow \\
\hline Dynamic & Clomiphene citrate challenge test (CCT) \\
& - Exogenous FSH ovarian response \\
& test (EFORT) \\
& Gonadotropin agonist stimulation \\
& test (GAST) \\
\hline
\end{tabular}




\section{CLINICAL CHARACTERISTICS}

Female age remains the predictor of first choice for estimating quality of ovarian reserve as the number of follicles irretrievably decreases with age (Fig. 1). Age of the woman is a simple way of obtaining information on the extent of ovarian reserve, both regarding quantity as well as quality. ${ }^{24}$ However, it is more important to identify those at a younger age with decreased ovarian reserve and those around the age of 41 years with adequate ovarian reserve. If such women are identified, it becomes easy to individualize the treatment protocol. It may also identify women for whom it is wise to refuse treatment or start treatment as early as possible before the reserves diminish. Moreover, poor response prediction by ORT may not always mean poor prognosis especially in a young patient. ${ }^{25}$

Menstrual cycle length (MCL) is supposed to be primarily determined by the rate and quality of follicular growth and, thus, the duration of the follicular phase; in general, menstrual cycles range from 21 to 35 days and exhibit a mean interval of 28 days. A gradual shortening in cycle length may initiate in the late 30s, in parallel with precocious higher serum levels of follicular stimulating hormone (FSH) and lower serum levels of inhibin $\mathrm{B}$ produced by a lesser number of antral follicles. ${ }^{26}$ In women with DOR, the follicular phase and/or the luteal phase may be shortened.

\section{BASAL FSH}

Basal serum FSH done on days 2, 3 and 4 of menstrual cycle increases with advancing age; however, the limitation is significant inter and intracycle variability as well as variability between assays. ${ }^{27} \mathrm{~A}$ change in the reference standard from human menopausal gonadotropin standard (International Reference Preparation [(IRP)-hMG] to the World Health Organization (WHO) second international standard (IRP 78/549) has changed FSH upper cut point from $25 \mathrm{IU}$ to $10 \mathrm{IU}$ (16.7 is corresponding to 25,10 corresponds to 15$).{ }^{28} \mathrm{FSH}$ is commonly used as a measure of ovarian reserve, and high values have been associated with both poor ovarian stimulation and failure to conceive. It has a high specificity of 83 to $100 \%$ and sensitivity varies widely from 10 to $80 \%$ for identifying women who will respond poorly. A recent study employing efficiency curves demonstrated $100 \%$ specificity for failure to achieve a live birth at FSH values above $18 \mathrm{IU} / \mathrm{l} .{ }^{29}$ The PPV of FSH for poor response to ovarian stimulation or failure to conceive is higher in older women. Besides FSH levels may fluctuate from cycle to cycle, in young women, prior to showing a raised plateau, the significance of which is poorly understood.
Although FSH rises with increasing reproductive age, it remains unknown whether high FSH levels in women of reproductive age predict an earlier onset of menopause. ${ }^{30}$ Consistently, elevated FSH concentrations confer a poor prognosis but a single elevated FSH value in women $<40$ years of age may not predict a poor response to stimulation or failure to achieve pregnancy. ${ }^{31}$

\section{ESTRADIOL}

Basal serum estradiol level on days 2, 3 or 4 of the menstrual cycle has poor inter- and intracycle reliability. Basal estradiol alone should not be used to screen for DOR. The test has value only as an aid to correct interpretation of a normal basal serum FSH value. An early rise in serum estradiol concentrations is a characteristic of reproductive aging and can lower an otherwise elevated basal FSH level into the normal range, causing a misinterpretation of the test. When the basal FSH concentration is normal but the estradiol level is elevated $(>60-80 \mathrm{pg} / \mathrm{ml})$ in the early follicular phase, there is limited evidence for an association with poor response, increased cancellation rates or lower pregnancy rates. $^{32}$

\section{ANTI-MULLERIAN HORMONE}

Anti-mullerian hormone (AMH) is produced by granulosa cells of preantral and small antral follicles beginning when the primordial follicles start developing and ending when they reach 2 to $6 \mathrm{~mm}$ in diameter (Fig. 2). The measurement of the size of primordial follicle pool is an indirect measure presuming that the size of small antral follicle pool is directly proportional to the primordial follicles. AMH is produced by follicles which are gonadotropin-independent and therefore remains relatively consistent within and between menstrual cycles in both normal young ovulating women

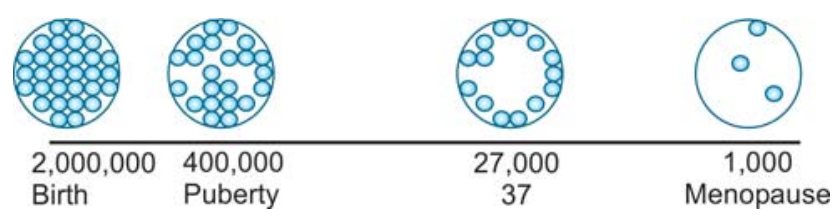

Fig. 1: Pattern of age-related decline in follicular number

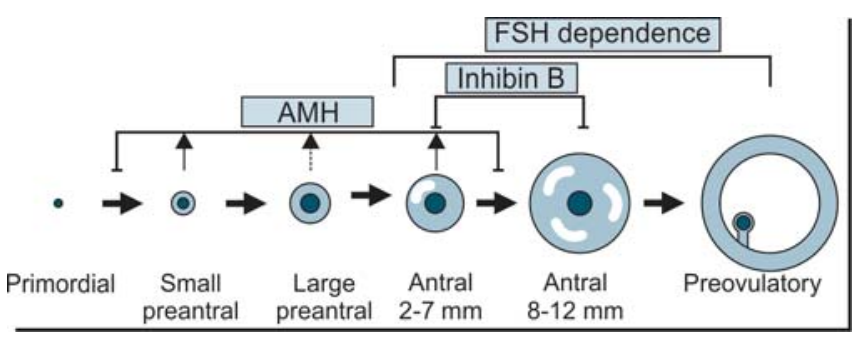

Fig. 2: Production of $A M H$ and inhibin $B$ by the follicles at different stages of growth 
and in women with infertility. ${ }^{33}$ The proposed clinical utility of $\mathrm{AMH}$ as an ovarian reserve test is mentioned in Table 4.

When applying AMH cut-points in clinical practice, clinicians must be very careful to determine that the assay used to measure $\mathrm{AMH}$ is the same as that used in the reference study population. Moreover, results can and do vary among different commercial laboratories using the same assay. Currently, AMH is tested by enzyme immunoassay (ELISA) and immunological assay of antibodies to AMH and shows considerable interassay variations. Lower AMH levels have been associated with, but do not necessarily predict poor response to ovarian stimulation, poor embryo quality and poor pregnancy outcomes in IVF. Studies that correlate different mean AMH levels with IVF outcomes do not provide useful AMH cut off for clinical care. ${ }^{34}$ However, combining AMH with AFC and maternal age improves its PPV.

The AMH cut off values for prediction of a poor response range from 0.5 to $1.1 \mathrm{ng} / \mathrm{ml}$ ( $\leq 3.6$ to $7.8 \mathrm{pmol} / \mathrm{L}) .{ }^{35}$ Similar to other markers, AMH should not be used to exclude couples from IVF. Women with a very poor prognosis should be carefully counselled regarding the potential difficulty in recruiting adequate follicles and hence cycle cancellation. These patients might be identified by serum $\mathrm{AMH}<0.1$ to $0.35 \mathrm{ng} / \mathrm{ml}^{36}$

$\mathrm{AMH}$ is a promising screening test and is likely more useful in the women at high risk for DOR than in women at low risk for DOR. Low AMH cut points are fairly specific for poor ovarian response to stimulation but not for pregnancy. Future studies of AMH as a screening test should incorporate larger numbers of subjects in a high risk or general IVF population. The use of $\mathrm{AMH}$ as a routine screening tool for DOR in a low-risk population is not recommended.

\section{INHIBIN B}

Inhibin $\mathrm{B}$ is not a reliable measure of ovarian reserve. Inhibin B levels rise with GnRH or FSH stimulation (the basis of

\section{Table 4: Proposed utility of AMH as an ORT}

1. Predicting both over- and poor-response in the controlled ovarian stimulation environment

2. Determining the most appropriate stimulation regimen

3. Pretreatment counseling for couples to make an appropriate and informed choice.

4. Predicting long-term fertility and guiding how long a woman can delay childbearing without facing the risk of reduced ovarian reserve

5. Predicting the age of menopause

6. Prediction of ovarian ageing in women prior to or following chemotherapy

7. Prediction of long-term fertility following ovarian surgery

8. Screening for polycystic ovaries dynamic tests of ovarian reserve) and therefore exhibit high intracycle variability. Inhibin B levels also vary significantly between menstrual cycles. Inhibin B is lower $(<40-45 \mathrm{pg} / \mathrm{ml})$ in poor responders than in women with a normal ovarian response to stimulation. Poor response is most commonly defined as $<3$ to 5 developing follicles, resulting in IVF cycle cancellation or as $<4$ retrieved oocytes. In population at high risk for DOR, PPV can be high. The large majority of studies have demonstrated that inhibin B does not discriminate between pregnancy and failure to conceive.

\section{ANTRAL FOLLICLE COUNT}

Antral follicle count (AIC) is the sum of antral follicles in both ovaries, as observed with transvaginal ultrasonography during the early follicular phase. Most studies have defined antral follicles as those measuring 2 to $10 \mathrm{~mm}$ in mean diameter in the greatest two-dimensional (2D) plane. Some have defined antral follicles as those measuring 3 to $8 \mathrm{~mm}$ in mean diameter. Antral follicle count has good intercycle reliability and interobserver reliability in experienced centers. ${ }^{37}$ A low AFC (range 3 to 10 total antral follicles) has been associated with (Fig. 3), but does not necessarily
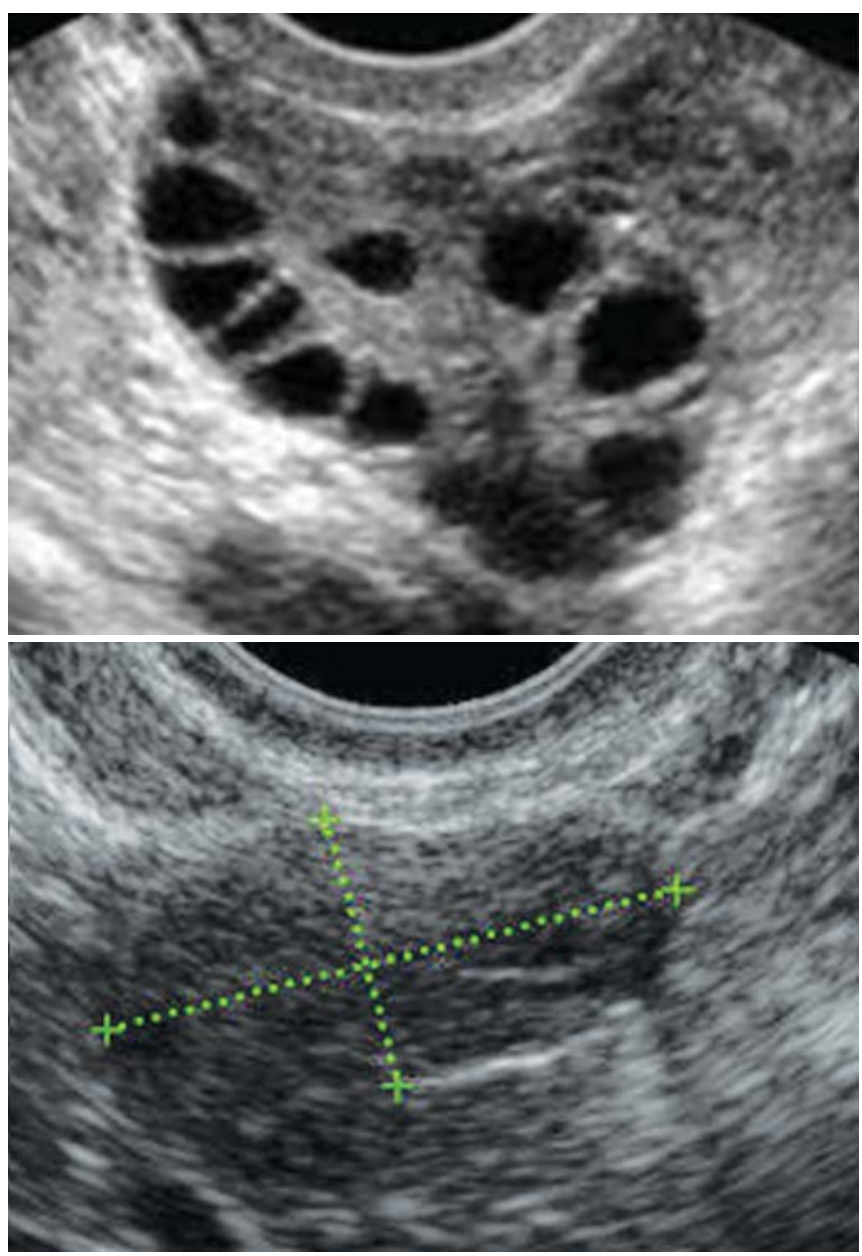

Fig. 3: Ultrasound picture of the antral follicle count (high AFC and low AFC) 
predict, poor response to ovarian stimulation and the failure to achieve pregnancy.

The high specificity of a low AFC makes the test useful for predicting poor ovarian response and treatment failure but its clinical utility is limited by its low sensitivity. Inter- and intraobserver variability also may be limiting, especially in centers having less expertise or lower quality ultrasound equipment.

\section{OVARIAN VOLUME}

Ovarian volume is calculated by measuring each ovary in three planes and using the formula for the volume of an ellipsoid (D1 × D2 × D3 × 0.52). Mean ovarian volume is the average volume of both ovaries in the same individual. Ovarian volume has limited reliability as an ovarian reserve test. Ovarian volume correlates with number of follicles and retrieved oocytes but not as well with pregnancy. Moreover, patients with ovarian pathology, including those with polycystic ovary syndrome, endometriomas, and large cysts cannot be considered. Antral follicle count is a better imaging test to screen for DOR than ovarian volume.

\section{OVARIAN BLOOD FLOW}

High pregnancy rate among women presenting with highly vascularized follicles in early follicular phase has been demonstrated. A recent meta-analysis assessed ovarian blood flow (OBF) as a predictor of IVF outcomes, but clinical value was unclear, because of different flow-derived predictors used in literature. Hence, ovarian vascular flow may not be used to determine inclusion of infertile couples in ART programs or to infer its results. ${ }^{38}$

\section{CLOMIPHENE CITRATE CHALLENGE TEST}

The clomiphene citrate challenge (CCCT) involves measurements of serum FSH before (cycle day 3) and after (cycle day 10) treatment with clomiphene citrate (100 mg daily, cycle days 5-9). Whereas rising inhibin B and estradiol levels derived from a growing cohort of ovarian follicles will suppress FSH in women with responsive ovaries, the smaller follicular cohorts that can be recruited in women with DOR will generate less inhibin B and estradiol, resulting in decreased negative feedback inhibition of FSH secretion and higher stimulated FSH concentrations. An elevated FSH concentration after clomiphene stimulation therefore suggests DOR.

In studies comparing the test performance of basal (cycle day 3) and stimulated (cycle day 10) FSH values, stimulated FSH levels have higher sensitivity but lower specificity than basal FSH concentrations. Compared to basal FSH and AFC, the clomiphene-stimulated day-10 FSH level does not clearly improve test accuracy for predicting poor ovarian response or pregnancy after IVF. In summary, basal measures of FSH may be preferable to the CCCT, unless one is using the test to purposely increase sensitivity. ${ }^{39}$

\section{OTHER DYNAMIC TESTS}

Gonadotropin agonist stimulation test (GAST) evaluates the change in estradiol levels on cycle days 2 and 3 following a subcutaneous administration of a gonadotropin-releasing hormone agonist (1 mg leuprolide acetate). The dose of the agonist causes a massive, temporary release of FSH and LH from the pituitary, which in turn increases estradiol production within a 24-hour time frame. A robust increase (twice the baseline value) or flare of estradiol in response to this stimulation is reflective of recruitable follicles in the early follicular phase which is in turn representative of OR. ${ }^{40}$

Exogenous FSH ovarian reserve test (EFORT) is based on the increase of E2 and inhibin B 24 hours after the administration of 300 IU of recombinant FSH (r-FSH) on cycle day 3, in order to determine the functional condition of the ovaries. Similar to CCCT and GAST, methodology for EFORT is far from uniform and should be avoided ${ }^{41}$ in determining ovarian reserve.

\section{COMBINATION OF TESTS}

Combination of endocrine and imaging tests improves the accuracy of poor response prediction. ${ }^{42}$ Predictive value of female age alone is quite limited and combinations of tests, like AFC and AMH, are sufficiently accurate to identify a poor responder even without knowing the age of the patient. Female age is highly related to prospects of pregnancy but it is inefficient in predicting who will become pregnant and who will not. AMH and AFC can be used for fine tuning of the prediction done by age (Fig. 4). However, few studies show that the addition of dynamic tests to AMH and AFC for prediction of ovarian reserve does not consistently improve specificity. Moreover, multiple tests add to the expense. Some have suggested that androgen deficiency may also be a test of ovarian reserve. However, no clear cut off levels are available.

\section{APPLICATION OF ORT IN CLINICAL PRACTICE}

ORT is very useful in prediction of response to stimulation. But, it is yet to be found that whether the application of these tests will be cost effective. They are not useful in

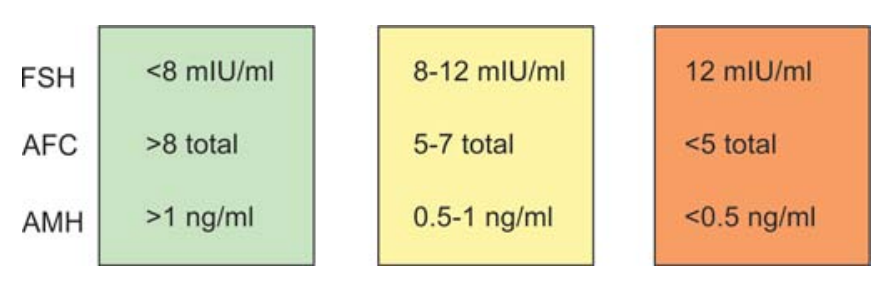

Fig. 4: $\mathrm{FSH} / \mathrm{AFC}$ and $\mathrm{AMH}$ in predicting ovarian reserve 
predicting pregnancy although they are used in clinical practice in combination with age to estimate response to ovarian stimulation. True challenge lies in the possibility of prediction in women with reduced reproductive lifespan at a stage in their lives when adequate action can be taken. Also if the age of menopause could be predicted, then the span between this age and the end of fertility could also be predicted, as it is estimated that a period of 10 years is fixed between the end of fertility and menopause. This can open a new way of individualized prevention of age-related infertility.

\section{MANAGEMENT}

Prevention of DOR is possible if it is due to an avoidable cause, such as smoking. DOR due to surgical causes can be prevented by careful surgical technique and strict adherence to principles of microsurgery. Laparoscopic ovarian drilling should be avoided in patients with low AMH even if the ovaries are polycystic in appearance. Patients undergoing chemotherapy or radiotherapy should be explained about the various options available for fertility preservation. Other strategies for patients undergoing chemoradiation are depot GnRH agonist therapy, oral contraceptives and transposition of ovaries. More recently, the use of apoptosis inhibitors like sphingosine-1-phosphate has been proposed. The various treatment regimens and adjuvants that have been proposed in patients with diminished ovarian reserves are enlisted in Table 5. However, there is no specific treatment to reverse DOR per se.

For clinical consideration, poor ovarian response (POR) or DOR according to the Bologna consensus ${ }^{43}$ indicates poor number of retrievable oocytes and is diagnosed by two of the following three criteria:

1. Advanced maternal age $\geq 40$ years or presence of other risk factors for POR.

2. Previous POR with $\leq 3$ oocytes retrieved in previous conventional stimulation.

3. Abnormal ovarian reserve tests $\mathrm{AFC}<5$ to 7 or $\mathrm{AMH}$ $<0.5$ to $1.1 \mathrm{ng} / \mathrm{ml}$.

The strategy of using high dose gonadotropins in ovarian stimulation in patients with likely poor response is not really

Table 5: Treatment regimens for patients with DOR

Treatment regimens:

1. High dose gonadotropins

2. Change of stimulation protocol

3. Natural cycle and modified natural cycle

4. LH supplementation

5. Androgens

6. Growth hormone

7. Aspirin

8. Ovum donation

9. Assisted hatching of much benefit, as the response is not dependant on the levels of gonadotropins but on the number of recruitable follicles. Moreover, there is a possibility of poor oocyte quality with use of higher dose of gonadotropins. ${ }^{44}$ Doubling the dose of gonadotropins during stimulation is not effective in improving the response and it goes well with the physiological fact that recruitment occurs not in the early follicular phase but in the late luteal phase of the previous cycle. ${ }^{45}$ Another strategy is to use high dose gonadotropins in the beginning and then step down in the second part of the cycle without compromising the cycle outcome. Increasing the starting dose to 300 IU may be effective but increase above 450 IU/day may have limited effectiveness. Administration of long acting gonadotropin, corifollitropin alfa has also been suggested.

Use of long agonist protocol may increase the ovarian unresponsiveness and increase the dose requirement of gonadotropins to two-fold due to decreased circulating levels and altered biological activity of endogenous gonadotropins, interference with follicular recruitment and direct ovarian inhibition. This led to introduction of mini dose GnRH agonist protocol in order to increase response especially in poor responders. Microdose flare regimens have been suggested to be of use in DOR, however, retrospective studies failed to find any benefit over long agonist protocols. ${ }^{46}$ The use of GnRH agonist short and ultrashort protocols also did not provide much benefit in terms of clinical pregnancy rates. GnRH antagonist protocols have been proposed to be of use in poor responders because it allows natural recruitment of follicles without any inhibitory effect.

Natural cycle, natural cycle modified with hCG, gonadotropins and antagonist have been proposed in patients with DOR. In a study done in comparison with microdose protocol, it was seen that natural cycle can be as effective as controlled ovarian stimulation, especially in young patients with DOR and higher implantation rate is seen. ${ }^{47}$ In patients with DOR, the implantation rates are similar in natural as well as stimulated cycles. Hence, natural cycle is preferred in view of low cost as well as more patient friendly approach.

LH supplementation during the stimulation protocol is known to be useful in poor responders as well as patients of advanced maternal age. Adequate steroidogenesis requires a certain threshold level of LH. It has been seen that sufficient steroidogenesis can occur even if $<1 \%$ of the $\mathrm{LH}$ receptors are occupied. Resting levels of $\mathrm{LH}$ are usually sufficient for adequate theca cell stimulation. Excessive levels of LH that cross the LH ceiling causes premature rise of progesterone which supresses aromatase activity and as a result causes atresia. Patients with DOR and PCOS are 
sensitive to fluctuations of LH levels. Patients with poor response to FSH stimulation especially of advanced age may benefit from LH supplementation from stimulation day 6 to 8 onward when the LH receptors are acquired. This could be because the number of functional LH receptors decreases with age. ${ }^{48,49}$

Androgens have a role in folliculogenesis in the early phase before the follicle becomes sensitive to gonadotropins. They inhibit the apoptosis and, in gonadotropin sensitive follicles, it enhances the FSH action. With increasing age, the levels of androgens decline. In downregulated women where the testosterone levels are low ( $<20 \mathrm{ng} / \mathrm{dl}$ ), higher FSH dose and longer days of stimulation are required. ${ }^{50} \mathrm{It}$ is suggested in patients of diminished ovarian reserve who are undergoing ART treatment that boosting androgens may increase the recruitment of follicles. This can be accomplished by LH and DHEA/testosterone supplementation. Beneficial effect of transdermal testosterone gel application for 21 days preceding ovarian stimulation in known poor responders has been shown to be of benefit in a RCT. There was increase in the number of oocytes, mature fertilized oocytes and good quality embryos compared to the controlled group. ${ }^{51}$

DHEA is supplemented in a dose of around $75 \mathrm{mg} /$ day and started 2 months prior and continued throughout stimulation. It has been summarized in a review by Gleicher that DHEA supplementation improves ovarian function, increases pregnancy chances and lowers aneuploidy and miscarriage rates. ${ }^{52}$ It has also been suggested that it is the first drug to beneficially effect ageing ovarian environment. Administration of DHEA for 2 to 3 months prior to ovarian stimulation has been recommended. In 25 women administered DHEA cycle outcome in IVF were compared pre and post DHEA administration. Results are shown in Table 6. We await further data on a larger cohort.

It is proposed that growth hormone $(\mathrm{GH})$ improves the outcome in poor responders by increasing the estradiol insulin growth factor-1 levels which promotes better developmental potential, enhanced nucleocytoplasmic maturation and DNA repair mechanisms. However, with the extra involved cost and insufficient data available, its role in DOR is not well established. Cochrane review done on the effectiveness of human growth hormone also confirmed this. ${ }^{54}$ Further studies are needed to better define the role and dose of human GH cotreatment. ${ }^{55}$

Low dose aspirin therapy has been demonstrated to enhance blood perfusion to multiple organ systems including the ovary. This is accomplished by greater inhibition of vasoconstricting prostaglandins than the vasodilating prostaglandins. In patients with poor response, aspirin is proposed to increase blood flow and responsiveness. In a RCT specifically done in poor responders, it was concluded that low dose aspirin failed to improve either blood flow or responsiveness. ${ }^{56}$ However, its use in patients with DOR is controversial and more trials are required in this regard.

Ovum donation from a young donor is the only option for management of DOR that is unresponsive to above therapies. However, it needs adequate counselling. The pregnancy rates in these women with ovum donation are as good as normal responders. ${ }^{57}$ Unfortunately, it is not widely available in many parts of the world due to cultural and social reasons.

Assisted hatching involves artificial breach in the zona pellucida and has been proposed to be a technique to improve the implantation and pregnancy rates. Breach is made without affecting the integrity of zona by proteolytic enzymes, acidified Tyrode's solution or laser. Assisted hatching is known to increase clinical pregnancy and multiple pregnancies in women with previous repeated implantation failures and in frozen thawed embryos. There is no benefit if it is applied to nonselected patients. ${ }^{58}$ However currently, there is insufficient evidence to recommend assisted hatching in women of advanced maternal age or poor ovarian response.

\section{ELECTIVE OOCYTE, EMBRYO AND OVARIAN TISSUE CRYOPRESERVATION TO DEFER CHILDBEARING}

It is widely accepted that there is progressive and rapid deterioration of the ovarian reserve in the females especially after the age of 35. Many career women may plan to defer childbearing due to professional/social reasons. For such a cohort, oocyte cryopreservation offers hope. This appears attractive but sufficient data is still not available on this

\begin{tabular}{lccc}
\multicolumn{4}{c}{ Table 6: Comparison of pre and post DHEA IVF cycles in 25 women with DOR } \\
& Pre DHEA & Post DHEA & $p$-value \\
\hline Cycle cancellation & $32 \%$ & $4.3 \%$ & 0.02 \\
Number of oocytes & $3.4 \pm 0.5$ & $4.4 \pm 0.5$ & $<0.05$ \\
Fertilized oocytes & $39 \%$ & $67 \%$ & $<0.001$ \\
Day 3 blastomeres & $3.4 \pm 0.4$ & $4.7 \pm 0.5$ & 0.01 \\
Number of embryos transferred & $1.4 \pm 0.2$ & $2.4 \pm 0.3$ & 0.005 \\
\hline
\end{tabular}


issue to recommend its widespread use. The factors like cost-effectiveness, safety and efficacy are not yet sufficiently assessed and women should not be encouraged on this issue as it may give them false hope and encourage them to defer childbearing. Success rates may be very low in women who plan to cryopreserve after 38 years of age. ${ }^{59}$

Patients who wish to utilize this technology should be carefully counselled about their age, clinic specific success rates, risk, cost, alternatives to this approach. They specifically need to be explained about the lower pregnancy and live birth rates with this procedure. Ovarian tissue cryopreservation and embryo cryopreservation are also options available to these patients. The use of ovarian tissue cryopreservation for later use has been explored in women undergoing anticancer treatment. Similarly, adolescent girls with Turner's syndrome still have follicles in their ovaries and could be candidates for ovarian cryopreservation. ${ }^{60}$

\section{POSTNATAL OOGENESIS}

It is a central dogma in reproductive biology that during the life of the individual there cannot be any increase in the number of primary oocytes beyond those originally laid down when the ovary was formed. However, a series of recent studies have challenged this dogma by showing regeneration of oocytes from putative germ cells in bone marrow and peripheral blood. ${ }^{61}$ These results not only triggered an enormous amount of interest among reproductive biologists but also a great deal of debate. While the number of commentaries on this topic continues to grow, recently oocytes and offspring were produced from female germ stem cells (FGSCs) in mice. ${ }^{62}$

In their study, FGSCs were isolated from mice and cultured for more than 6 months. Then these FGSCs were infected with green fluorescent protein (GFP) virus and transplanted into the ovaries of chemotherapy-sterilized mice. Transplanted cells underwent oogenesis and the mice produced offspring that had the GFP transgene. These results provided compelling evidence for the existence of FGSCs in adult mammalian females which, at least under the experimental conditions, are fully capable of generating oocytes that can be fertilized and yield viable offspring.

Currently, it is not known that the biological significance of these germ cells in relation to ovarian function and failure, whether the reported isolation of FGSCs from adult mouse ovaries is a reproducible observation or whether there are similar germ stem cells in the human ovary.

The potential implications of these findings for human reproduction could be significant not only for the preservation of the fertile status but also for the prevention of the diverse spectrum of health problems that emerge in women after depletion of ovarian reserve, which is progressive and irreversible with aging, and accelerated and premature in patients who are exposed to gonadotoxic chemotherapy and radiation regimens.

\section{CONCLUSION}

Ovarian reserve testing predictes the magnitude of ovarian response to ovarian stimulation but not the cycle fecundity. Female fertility declines with age but it is difficult to predict the pace of reproductive decline in an individual woman. Maintenance of regular cycles till the fertility potential has reduced to almost zero makes many females unaware of this phenomenon.

Ovarian reserve testing is very useful in patients at high risk of DOR as PPV will be high. True challenge lies in developing tests that predict DOR in a low risk population so that women can plan their reproductive careers. If DOR is diagnosed in women with reduced reproductive lifespan at a stage in their lives when adequate action can be taken, the challenge can be resolved. Prevention of further ovarian damage in patients with DOR can be avoided by cessation of smoking, careful surgical technique during ovarian surgery and strict adherence to principles of microsurgery and avoiding ovarian drilling and diathermy. Patients undergoing chemotherapy or radiotherapy should be explained about the various options available for fertility preservation. Lack of a definitive treatment strategy makes management of DOR a challenge for the reproductive specialist. Elective social oocyte cryopreservation and use of stem cells are promising modalities for the future.

\section{REFERENCES}

1. Stephen EH, Chandra A. Declining estimates of infertility in the United States: 1982-2002. Fertil Steril 2006;86:516-523.

2. Van Noord-Zaadstra BM, Looman CWN, Alsbach H, et al. Delaying childbearing: effect of age on fecundity and outcome of pregnancy. BMJ 1991;302:1361-1365.

3. Cooper AR, Baker VL, Sterling EW, Ryan ME, Woodruff TK, Nelson LM. The time is now for a new approach to primary ovarian insufficiency. Fertil Steril 2012;95:1890-1897.

4. Taylor AE. Systemic adversities of ovarian failure. J Soc Gynecol Investig 2001;8:S7-S9.

5. Snowdon DA, Kane RL, Beeson WL, Burke GL, Sprafka JM, Potter J, Iso H, Jacobs DR Jr, Phillips RL. Is early natural menopause a biologic marker of health and aging? Am J Public Health 1989;79:709-714.

6. Beerendonk CC, Braat DD. Present and future options for the preservation of fertility in female adolescents with cancer. Endocr Dev 2005;8:166-175.

7. Turner HH. A syndrome of infantilism, congenital webbed neck, and cubitus valgus. Endocrinology 1938;23:566.

8. Mattison DR, Donald R, Plowchalk DR, David R, Meadows MJ, Miller MM, Malek A, London S. The effect of smoking on oogenesis, fertilization and implantation. Semin Reprod Endocrinol 1989;7:291-304.

9. El-Nemr A, Al-Shawaf T, Sabatini L, Wilson C, Lower AM, Grudzinskas JG. Effect of smoking on ovarian reserve and ovarian stimulation in in vitro fertilization and embryo transfer. Hum Reprod 1998;13:2192-2198. 
10. Barbieri RL, McShane PM, Ryan KJ. Constituents of cigarette smoke inhibit human granulosa cell aromatase. Fertil Steril 1986;46:232-236.

11. Pron G, Mocarski E, Bennett J, Vilos G, Common A. Vanderburgh. Pregnancy after uterine artery embolization for leiomyomata: The Ontario Multicenter Trial. Obstet Gynecol 2005;105:67-76.

12. Nelson LM, Anasti JN, Flack MR. Premature ovarian failure. In: Adashi EY, Rock JA, Rosenwaks Z, editors. Reproductive endocrinology, surgery and technology. Philadelphia: Lippincott-Raven 1996;1393-1410.

13. Rebar RW, Connolly HV. Clinical features of young women with hyper-gonadotropic amenorrhea. Fertil Steril 1990;53:804-810.

14. Forges T, Monnier-Barbarino P. Premature ovarian failure in galactosaemia: pathophysiology and clinical management. Pathol Biol (Paris) 2003;51:47-56.

15. Davis OK, Ravnikar VA. Ovulation induction with clomiphene citrate in a woman with premature ovarian failure. A case report. J Reprod Med 1988;33:559-562.

16. Morrison JC, Givens JR, Wiser WL, Fish SA. Mumps oophoritis: a cause of premature menopause. Fertil Steril 1975;26:655-659.

17. Dar P, Sachs GS, Strassburger D, Bukovsky I, Arieli S. Ovarian function before and after salpingectomy in artificial reproductive technology patients. Hum Reprod 2000;15:142144 (level II-3).

18. Strandell A, Lindhard A, Waldenstrom U, Thorburn J. Prophylactic salpingectomy does not impair the ovarian response in IVF treatment. Hum Reprod 2001;16:1135-1139 (level I).

19. Sharara FI, Seifer DB, Flaws JA. Environmental toxicants and female reproduction. Fertil Steril 1998;70:613-622.

20. Whitney EA, Layman LC, Chan PJ, Lee A, Peak DB, McDonough PG. The follicle-stimulating hormone receptor gene is polymorphic in premature ovarian failure and normal controls. Fertil Steril 1995;64:518-524.

21. Morita Y, Tilly JL. Oocyte apoptosis: like sand through an hourglass. Dev Biol 1999;213:1-17.

22. Fasouliotis SJ, Simon A, Laufer N. Evaluation and treatment of low responders in assisted reproductive technique: a challenge to meet. J Assist Reprod Genet 2000;17:357-373.

23. van der Gaast MH, Eijkemans MJ, van der Net JB, de Boer EJ, Burger CW, van Leeuwen FE, Fauser BC, Macklon NS. Optimum number of oocytes for a successful first IVF treatment cycle. Reprod Biomed Online 2006;13:476-480.

24. Templeton A, Morris JK, Parslow W. Factors that affect outcome of in vitro fertilization. Lancet 1996;348:1402-1406.

25. Lashen H, Ledger W, Lopez-Bernal A, Barlow D. Poor responders to ovulation induction: is proceeding to in vitro fertilization worthwhile? Hum Reprod 1999;14:964-969.

26. Perloe M, Levy DP, Sills ES. Strategies for ascertaining ovarian reserve among women suspected of subfertility. Int J Fertil Womens Med 2000;45:215-224.

27. Kwee J, Schats R, McDonnell J, Lambalk CB, Schoemaker J. Intercycle variability of ovarian reserve tests: results of a prospective randomized study. Hum Reprod 2004;19:590-595.

28. Esposito MA, Coutifaris C, Barnhart KT. A moderately elevated day 3 FSH concentration has limited predictive value, especially in younger women. Hum Reprod 2002;17:118-123.

29. Scott RT Jr, Elkind-Hirsch KE, Styne-Gross A, Miller KA, Frattarelli JL. The predictive value for in vitro fertility delivery rates is greatly impacted by the method used to select the threshold between normal and elevated basal follicle-stimulating hormone. Fertil Steril 2008;89:868-878.
30. Soules MR, Sherman S, Parrott E, Rebar R, Santoro N, Utian W, et al. Executive summary: stages of Reproductive Aging Workshop (STRAW). Fertil Steril 2001;76:874-878.

31. Roberts JE, Spandorfer S, Fasouliotis SJ, Kashyap S, Rosenwaks Z. Taking a basal follicle-stimulating hormone history is essential before initiating in vitro fertilization. Fertil Steril 2005;83:37-41.

32. Smotrich DB, Widra EA, Gindoff PR, Levy MJ, Hall JL, Stillman RJ. Prognostic value of day 3 estradiol on in vitro fertilization outcome. Fertil Steril 1995;64:1136-1140.

33. Fanchin R, Taieb J, Lozano DH, Ducot B, Frydman R, Bouyer J. High reproducibility of serum anti-mullerian hormone measurements suggests a multi-staged follicular secretion and strengthens its role in the assessment of ovarian follicular status. Hum Reprod 2005;20:923.

34. van Rooij IA, Broekmans FJ, te Velde ER, et al. Serum antimullerian hormone levels: a novel measure of ovarian reserve. Hum Reprod 2002;17:3065-3071.

35. La Marca A, Sighinolfi G, Radi D, Argento C, Baraldi E, Artenisio AC, Stabile G, Volpe A. Anti-mullerian hormone $(\mathrm{AMH})$ as a predictive marker in assisted reproductive technology (ART). Hum Reprod Update 2010;16:113-130.

36. Ferraretti AP, La Marca A, Fauser BC, et al. ESHRE consensus on the definition of "poor response 'to ovarian stimulation for in vitro fertilization: the bologna criteria. Hum Reprod 2011;26: 1616-1624.

37. McIlveen M, Skull JD, Ledger WL. Evaluation of the utility of multiple endocrine and ultrasound measures of ovarian reserve in the prediction of cycle cancellation in a high-risk IVF population. Hum Reprod 2007;22:778-785.

38. Gibreel A, Maheshwari A, Bhattacharya S, Johnson NP. Ultrasound tests of ovarian reserve: a systematic review of accuracy in predicting fertility outcomes. Human Fertility 2009;12(2):95-106.

39. Hendriks DJ, Mol BW, Bancsi LF, te Velde ER, Broekmans FJ. The clomiphene citrate challenge test for the prediction of poor ovarian response and non-pregnancy in patients undergoing in vitro fertilization: a systematic review. Fertil Steril 2006;86: 807-818.

40. Carvalho B, Sobrinho DB, Vieire AD. Ovarian reserve assessment for infertility investigation. ISRN Obstet Gynecol 2012;2012:576385. doi: 10.5402/2012/576385.Epub 2012 Jan 26.

41. Maheshwari A, Gibreel A, Bhattacharya S, Johnson NP. Dynamic tests of ovarian reserve: a systematic review of diagnostic accuracy. Reproductive BioMedicine Online 2009;18(5):717-734.

42. Broekmans FJ, Kwee J, Hendriks DJ, Mol BW, Lambalk CB. A systematic review of tests predicting ovarian reserve and IVF outcome. Hum Reprod Update 2006;12:685-718.

43. Ferra retti AP, La Marca A, Fauser BC, et al. ESHRE consensus on the definition of poor response to ovarian stimulation for in vitro fertilization: the bologna criterion. Hum Reprod 2011;26: 1616-1624.

44. Ben Rafael Z, Benadiva CA, Ausmanas M, et al. Dose of human menopausal gonadotropin influences the outcome of an in vitro fertilization. Fertil Steril 1987;48:964-968.

45. Van Hooff MH, Alberda AT, Huisman GJ, Zeilmaker GH, Leerentveld RA. Doubling the dose of human menopausal gonadotropin in course of in vitro fertilization treatment cycle in poor responder: a randomized study. Human Reprod 1993;8: 369-373. 
46. Leondires MP, Escalpes M, Segars JH, Scott RT Jr, Miller BT. Microdose follicular phase gonadotropin releasing hormone agonist compared with luteal phase agonist for ovarian stimulation in in vitro fertilization. Fertil Steril 1999;72: 1018-1023.

47. Morgia F, Sbracia M, Schimberni M, et al. A controlled trial of natural cycle versus microdose GnRH flare cycles in poor responders undergoing invitro fertilization. Fertil Steril 2004;81: 1542-1547.

48. Vikho KK, Kujansuu E, Morsky P, Huhtaniemi I, Punnonen R. Gonadotropin and gonadotropin receptors during the perimenopause. Eur J Endocrinol Metab 2003;88:3327-3332.

49. Mochtar MH, Van der V, Ziech M, van Wely M. Recombinant luteinizing hormone (rLH) for controlled ovarian hyperstimulation in assisted reproductive cycles. Cochrane Database Syst Rev 2007:CD005070.

50. Frattarelli JL, Peterson EH. Effect of androgen levels on in vitro fertilization cycles. Fertil Steril 2004;81:1713-1714.

51. Kim CH, Howles CM, Lee HA. The effect of transdermal testosterone gel pre-treatment on controlled ovarian stimulation and IVF outcome in poor responders. Fertil Steril 2011;95: 679-683.

52. Gleicher N, Barad DH. DHEA supplementation in diminished ovarian reserve. Reprod Biol Endocrinol 20011;9:67.

53. Sunkara SK, Pundir J, Khalaf Y. Effect of androgen supplementation or modulation on ovarian stimulation outcome in poor responders: a meta-analysis. Reproductive Biomed Online 2011; 22:545-555.

54. Kotarba D, Kotarba J, Hughes E. Growth hormone for in vitro fertilization. Cochrane Database Syst Review 2000:CD000099.

55. De Ziegler D, Streuli I, Meldrum DR, Chapron C. The value of growth hormone suppliments in ART for poor ovarian responders. Fertil Steril 2011;96:1069-1076.

56. Lok IH, Yip SK, Cheung LP, Yin Leung PH, Haines CJ. Adjuvant low-dose aspirin therapy in poor-responders undergoing IVF: a prospective double blind placebo controlled trial. Fertil Steril 2004;81:556-561.
57. Shanbhag S, Aucott L, Bhattacharya S, Hamilton MA, McTavish AR. Interventions for poor responders to controlled ovarian hyperstimulation $(\mathrm{COH})$ in in vitro fertilization. Coch Database Syst Review 2007;(1):CD004379. Review.

58. Martins WP, Rocha IA, Ferriani RA, Nastri CO. Assisted hatching of human embryos: a systematic review and metaanalysis of randomised controlled trials. Hum Reprod Update 2011:17;248-253.

59. Borini A, Levi Setti PE, Anserini P, De luca R, De Santis L, Porcu E, La Sala GB, Ferraretti A, Bartolotti T, Coticchio G, et al. Multicentre observational study on slow cooling oocyte cryopreservation: clinical outcome. Fertil Steril 2010;94:1662-1668.

60. Hreinsson JG, Otala M, Fridstrom M, Borgstrom B, Rasmussen C, Lundqvist M, Tuuri T, Simberg N, Mikkola M, Dunkel L, et al. Follicles are found in the ovaries of adolescent girls with Turner's syndrome. J Clin Endocrinol Metab 2002;87:3618-3623.

61. Lee HJ, Selesniemi K, Niikura Y, Niikura T, Klein R, Dombkowski DM, Tilly JL. Bone marrow transplantation generates immature oocytes and rescues long-term fertility in a preclinical mouse model of chemotherapy-induced premature ovarian failure. J Clin Oncol 2007;22:3198-3204.

62. Zou K, Yuan Z, Yang Z, Luo H, Sun K, Zhou L, Xiang J, Shi L, Yu Q, Zhang Y, et al. Production of offspring from a germline stem cell line derived from neonatal ovaries. Nat Cell Biol 2009;5:631-636.

\section{ABOUT THE AUTHORS}

\section{Mandeep Kaur (Corresponding Author)}

Fellow, Department of Reproductive Medicine, Bangalore Assisted Conception Centre, Bengaluru, Karnataka, India, Phone: 08884838538 e-mail: docmandeep80@gmail.com

\section{Mala Arora}

Director, Noble IVF Centre, Faridabad, Haryana; Consultant, Fortis La Femme, Greater Kailash, New Delhi, India 